ESAIM: M2AN 48 (2014) 475-491

DOI: $10.1051 / \mathrm{m} 2 \mathrm{an} / 2013116$
ESAIM: Mathematical Modelling and Numerical Analysis

www.esaim-m2an.org

\title{
A MULTISCALE ENRICHMENT PROCEDURE FOR NONLINEAR MONOTONE OPERATORS
}

\author{
Y. Efendiev ${ }^{1,2}$, J. Galvis ${ }^{3}$, M. Presho ${ }^{1}$ And J. Zhou ${ }^{1}$
}

\begin{abstract}
In this paper, multiscale finite element methods (MsFEMs) and domain decomposition techniques are developed for a class of nonlinear elliptic problems with high-contrast coefficients. In the process, existing work on linear problems [Y. Efendiev, J. Galvis, R. Lazarov, S. Margenov and J. Ren, Robust two-level domain decomposition preconditioners for high-contrast anisotropic flows in multiscale media. Submitted.; Y. Efendiev, J. Galvis and X. Wu, J. Comput. Phys. 230 (2011) 937-955; J. Galvis and Y. Efendiev, SIAM Multiscale Model. Simul. 8 (2010) 1461-1483.] is extended to treat a class of nonlinear elliptic operators. The proposed method requires the solutions of (small dimension and local) nonlinear eigenvalue problems in order to systematically enrich the coarse solution space. Convergence of the method is shown to relate to the dimension of the coarse space (due to the enrichment procedure) as well as the coarse mesh size. In addition, it is shown that the coarse mesh spaces can be effectively used in two-level domain decomposition preconditioners. A number of numerical results are presented to complement the analysis.
\end{abstract}

Mathematics Subject Classification. 35J60, 65N30.

Received 29 July, 2013.

Published online March 11, 2014.

\section{INTRODUCTION}

Many fundamental modeling problems in engineering and physics exhibit multiscale behavior. In particular, the partial differential equations which are used to describe the physical nature of such systems often involve coefficients which vary over many length scales. Many of these problems have high contrast coefficients and nonlinearities (e.g., Forchheimer flow, nonlinear elasticity, etc.) which make the development of multiscale methods increasingly challenging. In this paper, we study the development of multiscale methods for nonlinear high-contrast elliptic equations.

In the past few decades, various multiscale solution techniques have been developed to capture the effects of small scales on a coarse grid $[1,2,11-14]$. In this paper, we follow the MsFEM framework where multiscale basis functions are constructed on a coarse grid. These coarse mesh basis functions are then incorporated into

Keywords and phrases. Generalized multiscale finite element method, nonlinear equations, high-contrast.

1 Department of Mathematics and Institute for Scientific Computation, Texas A \& M University, College Station, TX 77843, USA. efendiev@math.tamu.edu; mpresho@math.tamu.edu; jzhou@math.tamu.edu

2 SRI-Center for Numerical Porous Media, King Abdullah University of Science and Technology (KAUST), Thuwal 23955-6900, Kingdom of Saudi Arabia. yalchin.efendiev@kaust.edu.sa

3 Departmento de matematics, Universidad Nacional de Colombia, Bogota D.C., Colombia. jcgalvisa@unal.edu.co 
a multiscale global formulation in order to compute the solution. In recent years MsFEMs have been extended to systematically enrich initial coarse spaces to converge to the fine-grid solution $[9,10]$. In particular, we would like to mention Generalized Multiscale Finite Element Method [7], where the authors propose a systematic procedure for constructing coarse spaces. It has also been shown that the use of these coarse spaces in twolevel domain decomposition methods yields robust preconditioners for the iterative procedure (see e.g., $[6,10]$ ). Extending these methods to nonlinear problems requires the development of generalized enrichment strategies in non-Hilbert setting and coupling algorithms which we address in this paper.

In this paper, we develop MsFEM and domain decomposition for a class of nonlinear monotone elliptic operators with high-contrast coefficients. The non-linearity of the operator presents additional difficulties that must be considered before constructing the enriched coarse spaces mentioned above. Of particular importance is the method we use to obtain the nonlinear eigenpairs which are used in the enrichment procedure. Literature on methods for the numerical computation of eigenpairs is often focused on linear eigen problems or on finding the eigenfunction corresponding to a single (e.g., the smallest or largest) eigenvalue $[4,16]$. However, we emphasize that the multiscale enrichment necessitates the use of a number of eigenpairs corresponding to the nonlinear operator where many nice properties enjoyed by linear eigen problems are no longer available. Since the characterization and method proposed in $[19,20]$ for finding multiple nonlinear eigenpairs cannot solve our problem, it is particularly important that we develop some new characterization and method for finding multiple nonlinear eigenpairs leading to solve our problem. Once the appropriate spaces are constructed we compare MsFEM with local spectral basis functions with MsFEM that uses linear basis functions. Our numerical results show that one can obtain more accurate solutions when local spectral basis functions are used. In particular, the highconductivity features are captured more precisely with local spectral basis functions that identify and separate the high-contrast regions.

For added breadth, we propose the use of these coarse spaces in two-level domain decomposition preconditioners. Our approaches borrow the main ideas for nonlinear iterative methods from $[3,5,15,17]$. The number of iterations required by domain decomposition preconditioners is typically affected by the contrast in the media properties, in particularly due to the channelized high-contrast features. With an appropriate choice of coarse spaces, one can show that the number of iterations is independent of contrast (see [10] for linear problems). In this paper, we extend the methods developed for linear problems to nonlinear problems. We would like to note that the computations of eigenvectors are expensive; however, this is an offline cost and one can re-use these basis functions for different right hand sides and boundary conditions.

In order to confirm our theoretical findings we present a number of numerical examples. In particular, we present the convergence results for MsFEM corresponding to the addition of more eigenvectors in the coarse space enrichment and a change in the coarse mesh size. Our numerical results are consistent with our theoretical findings. In particular, we show that the convergence behaves as $H^{\gamma} / \Lambda^{*}$, where $H$ is the coarse mesh size and $\Lambda^{*}$ is the smallest eigenvalue such that the corresponding eigenvector is not included in the coarse space. The proof of this fact makes several assumptions which are formulated in Appendix. For two-level domain decomposition preconditioners designed in the paper, we show that the number of iterations is independent of the contrast.

The rest of the paper is organized as follows. In Section 2 we introduce the model problem as the motivation for the solution technique we consider. In Section 3 we describe the non-linear eigenvalue problem and describe the proposed coarse-grid solution technique. Section 4 is devoted to a detailed explanation of the eigenvalue computation, and in Section 5 we address two-level solvers. A variety of numerical results are presented in Section 6, and we offer some concluding remarks in Section 7.

\section{Preliminaries AND MOtivation}

We consider $u \in W_{0}^{1, p}(D), p \geq 2$,

$$
-\operatorname{div}(\kappa(x, \nabla u))=f
$$


where $\kappa=\left(\kappa_{i}\right)$. We assume that $\kappa(x, \xi)$ is monotone on $\xi \in \mathbb{R}^{d}$ and satisfies

$$
\begin{array}{r}
\left|\kappa\left(\cdot, \xi_{1}\right)-\kappa\left(\cdot, \xi_{2}\right)\right| \leq C\left(1+\left|\xi_{1}\right|+\left|\xi_{2}\right|\right)^{p-1-\alpha}\left|\xi_{1}-\xi_{2}\right|^{\alpha} \\
\left(\kappa\left(\omega, \xi_{1}\right)-\kappa\left(\omega, \xi_{2}\right)\right) \cdot\left(\xi_{1}-\xi_{2}\right) \geq C\left(1+\left|\xi_{1}\right|+\left|\xi_{2}\right|\right)^{p-\beta}\left|\xi_{1}-\xi_{2}\right|^{\beta} .
\end{array}
$$

For simplicity of the analysis we assume $\beta=p$ and $\kappa(\cdot, 0)=0$. Under these conditions, the solution exists and is unique [21].

The corresponding variational formulation is to find $u \in W_{0}^{1, p}(D)$ such that

$$
\int_{D} \kappa(x, \nabla u) \cdot \nabla v=\int_{D} f v, \text { for all } v \in W_{0}^{1, q}(D) .
$$

One can write a corresponding minimization problem where the solution can be thought as

$$
\inf _{u \in W_{0}^{1, p}(D)} \widetilde{F}(u) \text { where } \widetilde{F}(u)=\int_{D} \Pi(x, \nabla u)-\int_{D} f u \text { and } \frac{\partial}{\partial \xi_{i}} \Pi(\cdot, \xi)=\kappa_{i}(\cdot, \xi) .
$$

We define an energy "norm" by the notation

$$
\|u\|_{V(D)}^{p}=\int_{D} \Pi(x, \nabla u) .
$$

Next, we describe the finite element approximation of the solution. We let $\mathcal{T}^{h}$ denote a fine triangulation, and denote by $V^{h}=V^{h}(D)$ the usual finite element discretization of piecewise linear continuous functions with respect to $\mathcal{T}^{h}$. We also let $V_{0}^{h}(D)$ denote the subset of $V^{h}(D)$ with vanishing values on $\partial D$. Similar notation, $V^{h}(\Omega), V_{0}^{h}(\Omega)$, is used for $\Omega \subset D$.

The discrete fine-scale problem is defined to find $u \in V^{h}$ such that

$$
u=\arg \min _{v \in V^{h}} \widetilde{F}(v)
$$

or $\left\langle\widetilde{F}^{\prime}(u), v\right\rangle=0$, for all $v \in V^{h}$, where $\widetilde{F}\left(u_{h}\right)=\int_{D}\left(\Pi\left(x, \nabla u_{h}\right)-f u_{h}\right)$ or

$$
\left\langle\widetilde{F}^{\prime}(u), v\right\rangle=\int_{D} \kappa\left(x, \nabla u_{h}\right) \nabla v_{h}-\int_{D} f v_{h}=0, \quad \text { for all } v \in V^{h} .
$$

Example. One of the main examples we will study is the heterogeneous $p$-Laplacian. If $\widetilde{F}(v)=\frac{1}{p} \int_{D} \kappa(x)|\nabla v|^{p}-$ $\int_{D} f u$, we have

$$
\left\langle\widetilde{F}^{\prime}(u), v\right\rangle=\int_{D} \kappa(x)|\nabla u|^{p-2} \nabla u \nabla v-\int_{D} f v .
$$

The corresponding differential equation is to find $u \in W_{0}^{1, p}(D)$ such that

$$
-\operatorname{div}\left(\kappa(x)|\nabla u|^{p-2} \nabla u\right)=f .
$$

We introduce the coarse triangulation $\mathcal{T}^{H}$ and assume that $\mathcal{T}^{h}$ is a refinement of $\mathcal{T}^{H}$. We denote by $\left\{y_{i}\right\}_{i=1}^{N_{v}}$ the vertices of the coarse mesh $\mathcal{T}^{H}$ and define respectively the neighborhood $\omega_{i}$ of the node $y_{i}$ and the neighborhood $\omega_{K}$ of the coarse element $K$ by

$$
\omega_{i}=\bigcup\left\{K_{j} \in \mathcal{T}^{H} ; \quad y_{i} \in \bar{K}_{j}\right\}, \quad \omega_{K}=\bigcup\left\{\omega_{j} \in \mathcal{T}^{H} ; \quad y_{j} \in \bar{K}_{j}\right\} .
$$

Throughout this paper, the notation $a \preceq b$ means that $a \leq C b$ where the constant $C$ is independent of the mesh size and the contrast (which we denote by the physical parameter $\eta$ ). The constant $C$ may depend on $p$ and some other geometrical parameters of $\mathcal{T}^{H}$. 


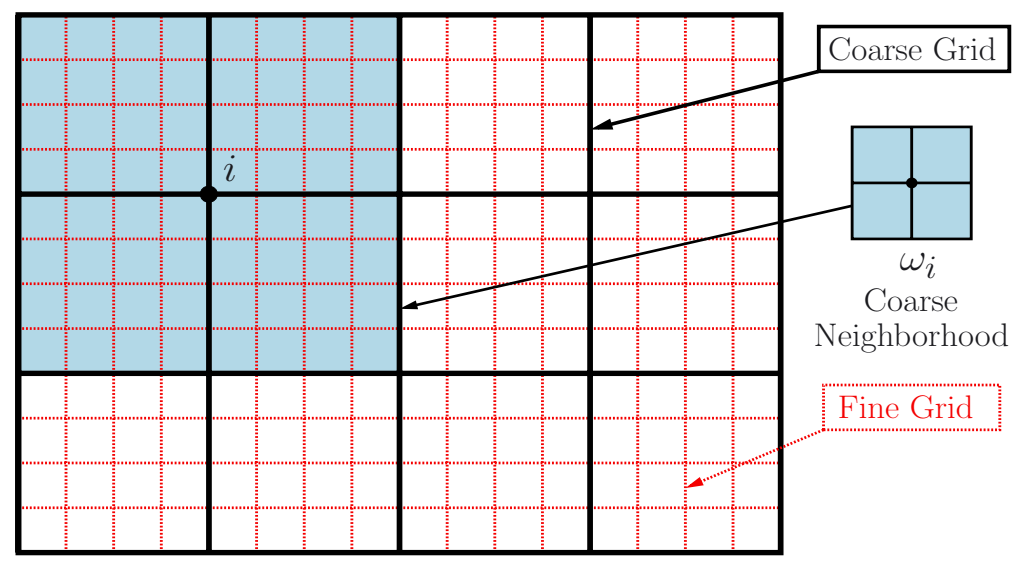

Figure 1. Coarse and fine grid.

\section{Multiscale technique}

In this section, we discuss our multiscale technique which involves the construction of a linear space of multiscale basis functions on a coarse grid. The construction starts with initial set of multiscale basis functions that will be enriched using a localized eigenvalue problems. More precisely, the dominant eigenmodes are multiplied by initial partition of unity functions $\left\{\chi_{i}\right\}_{i=1}^{N_{v}}$ that are subordinated to the covering $\left\{\omega_{i}\right\}_{i=1}^{N_{v}}$.

\subsection{Initial partition of unity}

First, we introduce some basic initial multiscale finite element spaces defined as one basis function per coarse node.

- A linear initial partition of unity, $\chi_{i}^{0}$ is defined as usual linear basis functions

- A multiscale initial partition of unity (with linear boundary conditions), $\chi_{i}^{\mathrm{ms}}$ is defined by

$$
-\operatorname{div}\left(\kappa\left(x, \nabla \chi_{i}^{\mathrm{ms}}\right)\right)=0 \text { in } K \in \omega_{i}, \quad \chi_{i}^{\mathrm{ms}}=\chi_{i}^{0} \text { in } \partial K, \text { for all } K \in \omega_{i} .
$$

- We can also use energy minimizing basis functions that are defined by

$$
\chi_{i}^{\mathrm{emf}}=\arg \min \sum_{i} \int_{\omega_{i}} \Pi\left(x, \nabla \chi_{i}\right)
$$

subject to $\sum_{i} \chi_{i}=1$ with $\operatorname{Supp}\left(\chi_{i}\right) \subset \omega_{i}, i=1, \ldots, N_{v}$ (see [18]).

\subsection{Eigenvalue problem and space enrichment}

In each patch $\omega_{i}$ we define

$$
G_{\chi}^{\omega_{i}}(u)=\sum_{k, \omega_{k} \cap \omega_{i} \neq 0}\left\|\chi_{k} u\right\|_{V\left(\omega_{i}\right)}^{p}, \quad \text { and } \quad G^{\omega_{i}}(u)=\|u\|_{V\left(\omega_{i}\right)}^{p} \text { for all } u \in V^{h}\left(\omega_{i}\right) .
$$

Next, we define

$$
\lambda^{\omega_{i}}(u)=\frac{G^{\omega_{i}}(u)}{G_{\chi}^{\omega_{i}}(u)} \text { for all } u \neq 0, u \in V^{h}\left(\omega_{i}\right) .
$$

To compute our multiscale space, we want to define a coarse space $V_{c}^{h}\left(\omega_{i}\right)$ (a space with a minimum dimension) such that for any $u \in V^{h}\left(\omega_{i}\right)$ there exists $u_{0} \in V_{c}^{h}\left(\omega_{i}\right)$ satisfying

$$
\lambda^{\omega_{i}}\left(u-u_{0}\right)>\lambda_{0} .
$$


A coarse space is constructed in the following way. For each patch $\omega_{i}$, we identify a spectral problem and dominant eigenvectors

$$
\psi_{1}^{\omega_{i}}, \ldots, \psi_{L_{i}}^{\omega_{i}} \text { and denote } V^{c}\left(\omega_{i}\right)=\operatorname{Span}\left\{\psi_{1}^{\omega_{i}}, \ldots, \psi_{L_{i}}^{\omega_{i}}\right\}
$$

The multiscale basis functions for the patch $\omega_{i}$ are constructed by

$$
V^{C}\left(\omega_{i}\right)=\operatorname{Span}\left\{\chi_{i}^{\omega_{i}} \psi_{1}^{\omega_{i}}, \ldots, \chi_{i}^{\omega_{i}} \psi_{L_{i}}^{\omega_{i}}\right\}
$$

Then, a coarse space is given by

$$
V^{C}=\bigcup_{\omega_{i}} V^{C}\left(\omega_{i}\right)
$$

and the corresponding coarse problem is defined by

$$
u=\arg \min _{v \in V^{C}} \widetilde{F}(v) .
$$

\subsubsection{Motivation of the eigenvalue problem}

We let $u \in V^{h}(D)$, and define an interpolant in $\omega_{i}, I^{\omega_{i}} u$, such that $I^{\omega_{i}} u \in \operatorname{Span}\left\{\psi_{1}^{\omega_{i}}, \ldots, \psi_{L_{i}}^{\omega_{i}}\right\}$ and $\lambda^{\omega_{i}}(u-$ $\left.I^{\omega_{i}} u\right)>\lambda_{0}$. Let $v$ be the approximation of $u$ over $D$, and defined as $v=\sum_{i} \chi_{i} I^{\omega_{i}} u$. Given $u$, we can write

$$
\|u-v\|_{V(D)}^{p} \preceq \sum_{i} G_{\chi}^{\omega_{i}}\left(u-I^{\omega_{i}} u\right) .
$$

The eigenvalue problem is motivated by the fact that we would like to bound $G_{\chi}^{\omega_{i}}\left(u-I^{\omega_{i}} u\right)$ by a term independent of $\chi$ and $I^{\omega_{i}} u$. In this paper, we will bound $G_{\chi}^{\omega_{i}}\left(u-I^{\omega_{i}} u\right)$ by $G^{\omega_{i}}\left(u-I^{\omega_{i}} u\right)$. The latter motivates the eigenvalue problem.

\section{Eigenvalue computation}

In this section we describe a solution technique for solving nonlinear eigenproblems which are motivated by the previous section. First, we let $V^{h}\left(\omega_{i}\right)$ be the space spanned by the basis functions of the finite element mesh $\mathcal{T}^{h}$ localized to a coarse neighborhood $\omega_{i}$. Now we assume the homogeneous condition $\kappa(\cdot, t \xi)=|t|^{p-1} \kappa(\cdot, \xi) \forall t \neq 0$. Then we have

$$
\begin{aligned}
\Pi(\cdot, \xi) & =\frac{1}{p} \kappa(\cdot, \xi) \cdot \xi, \quad G^{\omega_{i}}(t u)=|t|^{p} G^{\omega_{i}}(u), \quad G_{\chi}^{\omega_{i}}(t u)=|t|^{p} G_{\chi}^{\omega_{i}}(u), \\
\left(G^{\omega_{i}}\right)^{\prime}(t u) & =|t|^{p-1}\left(G^{\omega_{i}}\right)^{\prime}(u), \quad\left(G_{\chi}^{\omega_{i}}\right)^{\prime}(t u)=|t|^{p-1}\left(G_{\chi}^{\omega_{i}}\right)^{\prime}(u), \quad \lambda^{\omega_{i}}(t u)=\lambda^{\omega_{i}}(u) \forall t \neq 0 .
\end{aligned}
$$

We denote $A_{S}\left(\omega_{i}\right)=\left\{v \in A\left(\omega_{i}\right): G_{\chi}^{\omega_{i}}(v)=1\right\}$ for all $A\left(\omega_{i}\right) \subset V^{h}\left(\omega_{i}\right)$. We define the smallest eigenvalue by $\lambda_{1}^{\omega_{i}}$ and the corresponding eigenvector $\psi_{1}^{*, \omega_{i}}=\arg \min _{u \in V_{S}^{h}\left(\omega_{i}\right)} \lambda^{\omega_{i}}(u)$ and $\lambda_{1}^{\omega_{i}}=\lambda^{\omega_{i}}\left(\psi_{1}^{*, \omega_{i}}\right)$.

We reiterate that the eigenvalue problem is used in order to systematically enrich our coarse solution space. As such, we formulate two important (in the context of multiscale enrichment) sets of inequalities below.

Main Problem: For a threshold $\lambda^{*, \omega_{i}}>\lambda_{1}^{\omega_{i}}>0$, find a minimum subspace (called coarse space) $V^{c}\left(\omega_{i}\right) \subset$ $V^{h}\left(\omega_{i}\right)$ such that

$$
C\left(\omega_{i}\right) G^{\omega_{i}}(u) \geq G^{\omega_{i}}\left(u-I^{\omega_{i}} u\right) \geq \lambda^{*, \omega_{i}} G_{\chi}^{\omega_{i}}\left(u-I^{\omega_{i}} u\right) \quad \text { for all } u \in V^{h}\left(\omega_{i}\right),
$$

where $I^{\omega_{i}} u \in V^{c}\left(\omega_{i}\right)$ and $C\left(\omega_{i}\right)>0$ depends on $p$, but is independent of $u$ and $\kappa$.

Sub-Problem: Given $\lambda^{*, \omega_{i}}>\lambda_{1}^{\omega_{i}}$, find a maximum subspace $U^{h}\left(\omega_{i}\right) \subset V^{h}\left(\omega_{i}\right)$ such that

$$
G^{\omega_{i}}(u) \geq \lambda^{*, \omega_{i}} G_{\chi}^{\omega_{i}}(u) \text { for all } u \in U^{h}\left(\omega_{i}\right) .
$$


In the simplified case when $p=2$ (a self-adjoint linear problem), the sub-problem becomes equivalent to finding the smallest eigenvalue $\lambda_{k}^{\omega_{i}} \geq \lambda^{*, \omega_{i}}$ to the linear eigenproblem

$$
\left(G^{\omega_{i}}\right)^{\prime}(u)=\lambda^{\omega_{i}}\left(G_{\chi}^{\omega_{i}}\right)^{\prime}(u)
$$

which can be characterized by the Rayleigh-Ritz method (RRM)

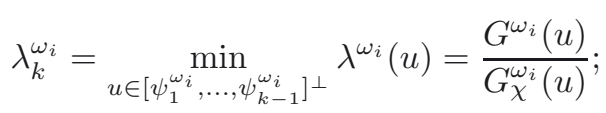

a standard orthogonal subspace minimization method (see e.g., [21]). It is important to note that in the case when $p>2,\left(G^{\omega_{i}}\right)^{\prime}(u)=\lambda^{\omega_{i}}\left(G_{\chi}^{\omega_{i}}\right)^{\prime}(u)$ is nonlinear and $\lambda_{k}^{\omega_{i}}$ obtained by RRM is not strictly optimal although the orthogonality $\perp$ is well-defined here.

To develop a new method, we first note that $\left(G^{\omega_{i}}\right)^{\prime}(u)=\lambda^{\omega_{i}}\left(G_{\chi}^{\omega_{i}}\right)^{\prime}(u)$ can be characterized by the Courant-Fischer-Weyl (CFW) max-min principle (see [21]). The sub-problem can be solved as to find the first $\lambda_{k}^{\omega_{i},-} \geq \lambda^{\omega_{i}, *}$ where

$$
\lambda_{k}^{\omega_{i},-}=\max _{A\left(\omega_{i}\right) \in \mathcal{S}_{k}\left(\omega_{i}\right)} \min _{u \in A\left(\omega_{i}\right)} \lambda^{\omega_{i}}(u), \quad k=2, \ldots, n,
$$

and $\mathcal{S}_{k}\left(\omega_{i}\right)$ denotes the set of all $k-1$ co-dimensional subspaces in $V^{h}\left(\omega_{i}\right)$. We let $A_{k}^{-}\left(\omega_{i}\right) \in \mathcal{S}_{k}\left(\omega_{i}\right)$ be such that

$$
\lambda_{k}^{\omega_{i},-}=\min _{u \in A_{k}^{-}\left(\omega_{i}\right)} \lambda^{\omega_{i}}(u)
$$

Then $\lambda_{k}^{\omega_{i},-}<\lambda_{k+1}^{\omega_{i},-}$ and the optimality can be stated by

$$
G^{\omega_{i}}(u) \geq \lambda_{k}^{\omega_{i},-} G_{\chi}^{\omega_{i}}(u) \text { for all } u \in A_{k}^{-}\left(\omega_{i}\right)
$$

where the inequality will be violated if $\lambda_{k}^{\omega_{i},-}$ is replaced by any $\lambda^{\omega_{i}}>\lambda_{k}^{\omega_{i},-}$. We note that CFW (4.4) was originally established for solving the linear problem $\left(G^{\omega_{i}}\right)^{\prime}(u)=\lambda^{\omega_{i}}\left(G_{\chi}^{\omega_{i}}\right)^{\prime}(u)$ (see e.g., [21]). For our problem we first establish the solvability of the nonlinear analogue in (4.4). The sub-problem solvability is stated in the following theorem.

Theorem 4.1. Sub-problem (4.4) is solvable.

Proof. We refer the interested reader to the Appendix.

We note that the max-min problem (see Eq. (4.4)) still cannot be numerically implemented, because we cannot cover all $k-1$ co-dimensional subspaces. Thus, we need to develop a numerically implementable maxmin characterization.

It can be shown that the solutions of

$$
\psi_{k}^{*, \omega_{i}}=\arg \max _{A\left(\omega_{i}\right) \in \mathcal{S}_{k}\left(\omega_{i}\right)} \min _{u \in A(\omega)} \lambda^{\omega_{i}}(u)=\arg \min _{u \in A_{k}\left(\omega_{i}\right)} \lambda^{\omega_{i}}(u),
$$

correspond to the critical points of $\lambda^{\omega_{i}}$, i.e.,

$$
\left(\lambda^{\omega_{i}}\right)^{\prime}\left(\psi_{k}^{*, \omega_{i}}\right)=0 \text { or }\left(G^{\omega_{i}}\right)^{\prime}\left(\psi^{*, \omega_{i}}\right)=\lambda^{\omega_{i}}\left(\psi_{k}^{*, \omega_{i}}\right)\left(G_{\chi}^{\omega_{i}}\right)^{\prime}\left(\psi_{k}^{*, \omega_{i}}\right),
$$

since

$$
\left(\lambda^{\omega_{i}}\right)^{\prime}\left(\psi_{k}^{*, \omega_{i}}\right) \equiv\left[G_{\chi}^{\omega_{i}}\left(\psi_{k}^{*, \omega_{i}}\right)\right]^{-1}\left[\left(G^{\omega_{i}}\right)^{\prime}\left(\psi_{k}^{*, \omega_{i}}\right)-\lambda^{\omega_{i}}\left(\psi_{k}^{*, \omega_{i}}\right)\left(G_{\chi}^{\omega_{i}}\right)^{\prime}\left(\psi_{k}^{1, \omega_{i}}\right)\right]=0 .
$$

Equivalently, $\left(\lambda^{\omega_{i}}\left(\psi_{k}^{*, \omega_{i}}\right), \psi_{k}^{*, \omega_{i}}\right)$ is a desired eigensolution of the nonlinear eigenproblem

$$
\left(G^{\omega_{i}}\right)^{\prime}(u)-\lambda^{\omega_{i}}\left(G_{\chi}^{\omega_{i}}\right)^{\prime}(u)=0
$$


To reduce the choices of max in (4.4) we consider RRM

$$
\psi_{k}^{0, \omega_{i}}=\arg \min _{u \in\left[\psi_{1}^{\omega_{i}}, \ldots, \psi_{k-1}^{\omega_{i}}\right] \perp \in \mathcal{S}_{k}\left(\omega_{i}\right)} \lambda^{\omega_{i}}(u)=\frac{G^{\omega_{i}}(u)}{G_{\chi}^{\omega_{i}}(u)}, k=1,2, \ldots
$$

for $p$-Laplacian (see (2.4)) and choose the inner product

$$
\langle u, v\rangle_{\kappa}=\int_{\omega_{i}}[\kappa(x) \nabla u(x) \cdot \nabla v(x)+\widetilde{\kappa}(x) u(x) v(x)] \mathrm{d} x .
$$

where $\widetilde{\kappa}=\kappa \sum_{i} H^{p}\left|\nabla \chi_{i}\right|^{p}$. We also denote

$$
\langle u, v\rangle=\int_{\omega_{i}} \kappa(x) u(x) v(x) \mathrm{d} x .
$$

Then $V^{c}\left(\omega_{i}\right)=\left[\psi_{1}^{\omega_{i}}, \ldots, \psi_{k-1}^{\omega_{i}}\right]$ represents a coarse space and

$$
G^{\omega_{i}}(u) \geq \lambda^{\omega_{i}}\left(\psi_{k}^{0, \omega_{i}}\right) G_{\chi}^{\omega_{i}}(u) \text { for all } u \in V^{c}\left(\omega_{i}\right)^{\perp}
$$

or

$$
G^{\omega_{i}}\left(u-I^{\omega_{i}} u\right) \geq \lambda\left(\psi_{k}^{0, \omega_{i}}\right) G_{\chi}^{\omega_{i}}\left(u-I^{\omega_{i}} u\right) \text { for all } u \in V^{h}\left(\omega_{i}\right) .
$$

But such $\lambda\left(\psi_{k}^{0, \omega_{i}}\right)$ is not optimal for $p>2$, and to improve it, we use RRM as a prediction and then a max-min method as a correction. The detailed steps of the nonlinear eigenproblem solution technique is presented in the algorithm below.

A Prediction-Correction Max-Min Method (PCMM): Set $k=2$.

Step 0. Let $\left(\mu_{m}^{\omega_{i}}, \psi_{m}^{\omega_{i}}\right)$ be the eigensolutions to

$$
\left\{\begin{array}{l}
-\operatorname{div}\left(\kappa(x) \nabla \psi^{\omega_{i}}\right)=\mu \kappa(x) \psi^{\omega_{i}}(x) \\
\frac{\partial \psi^{\omega_{i}}}{\partial n}=0, \quad x \in \partial \omega_{i} ; \quad\left\|\psi_{m}\right\|=1 .
\end{array}\right.
$$

Step 1. (Prediction) Do

$$
\psi_{k}^{\prime, \omega_{i}}=\arg \min _{u \in\left[\psi_{1}^{\omega_{i}}, \ldots, \psi_{k-1}^{\omega_{i}}\right]_{S}^{\perp} \in \mathcal{S}_{k}\left(\omega_{i}\right)} \lambda^{\omega_{i}}(u) .
$$

Step 2. (Correction) With the initial guess $\psi_{k}^{\prime, \omega_{i}}$, i.e., write $\psi_{k}^{\prime, \omega_{i}}=\psi_{k\left[\psi_{1}^{\prime}, \ldots, \omega_{k}^{\omega_{i}}\right]}^{\prime \omega_{i}}+\psi_{k\left[\psi_{1}^{\omega_{i}, \ldots, \psi_{k}} \psi_{k}^{\omega_{i}}\right]^{\perp}}^{\prime, \omega_{i}}$ and use $\psi_{k\left[\psi_{1}^{\omega_{i}}, \ldots, \psi_{k}^{\left.\omega_{i}\right]}\right.}^{\prime, \omega_{i}} \in\left[\psi_{1}^{\omega_{i}}, \ldots, \psi_{k}^{\omega_{i}}\right]$ as an initial guess do

$$
\psi_{k}^{0, \omega_{i}}=\arg \max _{u \in\left[\psi_{1}^{\omega_{i}}, \ldots, \psi_{k}^{\omega_{i}}\right]_{S}} \min _{v \in\left[u,\left[\psi_{1}^{\omega_{i}}, \ldots, \psi_{k}^{\omega_{i}}\right]^{\perp}\right]_{S} \in \mathcal{S}_{k}\left(\omega_{i}\right)} \lambda^{\omega_{i}}(v)
$$

and denote

$$
\psi_{k}^{*, \omega_{i}}=\arg \min _{v \in\left[\psi_{k}^{0, \omega_{i}},\left[\psi_{1}^{\omega_{i}}, \ldots, \psi_{k}^{\omega_{i}}\right]^{\perp}\right]_{S}} \lambda^{\omega_{i}}(v) .
$$

Step 3. (Check)

If $\lambda^{\omega_{i}}\left(\psi_{k}^{*, \omega_{i}}\right) \geq \lambda_{0}^{\omega_{i}}>\lambda^{\omega_{i}}\left(\psi_{k-1}^{*, \omega_{i}}\right) \quad$ (succeeded) go to Step 4;

Else set $k=k+1$ and check if $k=n$ (failed) stop, else go to Step 1 .

Step 4. We have optimality of solutions obtained by the method (see Eqs. (4.11), (4.12), and (4.13)) 
Upon completion of the algorithm we denote $V^{c}\left(\omega_{i}\right)=\left[\psi_{k}^{0, \omega_{i}},\left[\psi_{1}^{\omega_{i}}, \ldots, \psi_{k}^{\omega_{i}}\right]^{\perp}\right]^{\perp}$. We note that reaching Step 4 of the PCMM algorithm yields the optimality conditions

$$
\begin{aligned}
G^{\omega_{i}}\left(\psi_{k}^{*, \omega_{i}}\right) & =\lambda^{\omega_{i}}\left(\psi_{k}^{*, \omega_{i}}\right) G_{\chi}^{\omega_{i}}\left(\psi_{k}^{*, \omega_{i}}\right), \psi_{k}^{*, \omega_{i}} \in\left[\psi_{k}^{0, \omega_{i}},\left[\psi_{1}^{\omega_{i}}, \ldots, \psi_{k}^{\omega_{i}}\right]^{\perp}\right], \\
\left(\lambda^{\omega_{i}}\right)^{\prime}\left(\psi_{k}^{*, \omega_{i}}\right) & =0 \\
G^{\omega_{i}}(u) & \geq \lambda^{\omega_{i}}\left(\psi_{k}^{*, \omega_{i}}\right) G_{\chi}^{\omega_{i}}(u) \quad \text { for all } u \in\left[\psi_{k}^{0, \omega_{i}},\left[\psi_{1}^{\omega_{i}}, \ldots, \psi_{k}^{\omega_{i}}\right]^{\perp}\right] \in \mathcal{S}_{k}\left(\omega_{i}\right),
\end{aligned}
$$

at least for all such $u$ close to $\psi_{k}^{*, \omega_{i}}$; and the above inequality will be violated if $\lambda^{\omega_{i}}\left(\psi_{k}^{*, \omega_{i}}\right)$ is replaced by any $\lambda^{\omega_{i}}>\lambda^{\omega_{i}}\left(u_{k}^{*, \omega_{i}}\right)$ or $\psi_{k}^{0, \omega_{i}}$ is replaced by any other $u \in\left[\psi_{1}^{\omega_{i}}, \ldots, \psi_{k}^{\omega_{i}}\right]$.

\section{Domain DeCOMPosition ITERATION FOR THE FINE-SCALE SOLUTION}

We will also use the coarse spaces constructed via the solution of local nonlinear eigenvalue problem in a two-level (nonlinear) domain decomposition method. We focus in Schwarz subspace minimization algorithms. We refer the interested reader to $[3,5,15,17]$ for more discussion on nonlinear domain decomposition methods.

In order to describe the two-level domain decomposition we introduce some notation. We use the overlapping decomposition generated by the coarse grid neighborhoods, i.e., the decomposition $\left\{\omega_{i}\right\}_{i=1}^{N}$. More general overlapping decomposition can be considered as well. We use $V^{C}\left(\omega_{i}\right)$, the set of finite element functions with support in $\omega_{i}$ and zero trace on the boundary $\partial \omega_{i}$. In general, one can use a general coarse space. We also denote by $R_{i}^{T}: V^{C}\left(\omega_{i}\right) \rightarrow V^{h}$ the extension by zero operator.

We define the local problems as follows. Find $P_{i}(u) \in V^{C}\left(\omega_{i}\right)$ such that

$$
P_{i}(u)=\arg \min _{v_{i} \in V^{C}\left(\omega_{i}\right)} \widetilde{F}\left(u-R_{i}^{T} v_{i}\right)
$$

or, equivalently for the cases considered in this paper,

$$
\left\langle\widetilde{F}^{\prime}\left(u-R_{i}^{T} P_{i} u\right), R_{i}^{T} z_{i}\right\rangle=0, \quad \text { for all } z \in V^{C}\left(\omega_{i}\right) .
$$

Note that if $u$ is the solution of the original problem, then $P_{i} u=0$. Starting with an initial guess $u_{0} \in V^{h}(D)$, we introduce the nonlinear one level subspace iteration defined by

$$
u_{n+1}=u_{n}+\sum_{i=1}^{N} \alpha_{i} R_{i}^{T} P_{i}\left(u_{n}\right)=u_{n}+P_{1 L}\left(u_{n}\right) .
$$

Here $\alpha_{i}, i=1, \ldots, N$ are constants such that $\sum_{i=1}^{N} \alpha_{i}=1$ and we introduced the notation $P_{1 L}(u)=$ $\sum_{i=1}^{N} \alpha_{i} R_{i}^{T} P_{i}\left(u_{n}\right)$. Note that, when $\widetilde{F}$ is linear, this iteration corresponds to the one level additive Schwarz solver. It is known that this solver is not robust with respect to the contrast (even for linear problems). To get more robust iterations a coarse problem is added. The nonlinear two level subspace iteration is

$$
u_{n+1}=u_{n}+\left(1-\alpha_{0}\right) P_{1 L}\left(u_{n}\right)+\alpha_{0} P_{0}(u)=u_{n}+P_{2 L}\left(u_{n}\right) .
$$

where $P_{0} u$ is the solution of the coarse problem (3.3) and $0<\alpha_{0}<1$.

Remark 5.1. When the resulting equation, $\left\langle\widetilde{F}^{\prime}(u), v\right\rangle$ is a linear equation. it is well known that the converge of the Schwarz methods can be accelerated, e.g., by using a preconditioned Krylov subspace method to solve the equation $P_{2 L}=0$ where the preconditioned operator corresponds to the operator $P_{2 L}=M_{2 L}^{-1} \widetilde{F}^{\prime}$ where $M_{2 L}^{-1}$ is the two level preconditioner. In the nonlinear case we can also accelerate the converge of the method by considering a (quasi-)Newton method for the equation

$$
P_{2 L}(u)=0 .
$$




\section{A FULLY RESOlVED METHOD AND ITS NUMERICAL RESUltS}

In this section, to verify and implement the proposed enrichment approach in Section 3, as well as the nonlinear eigenpair computations described in Section 4, we consider multiscale $p$-Laplacian equation with the high contrast in the coefficients

$$
-\operatorname{div}\left(\kappa(x)|\nabla u|^{p-2} \nabla u\right)=f \quad \text { for } x \in D,
$$

where $\kappa(x)$ is a high contrast (i.e., $\kappa_{\max }(x) / \kappa_{\min }(x)=\eta$ where $\eta$ is large), heterogeneous coefficient. We note that this is a special case of $(2.3)$ where

$$
\left\langle\widetilde{F}^{\prime}(u), v\right\rangle=\int_{D} \kappa(x)|\nabla u|^{p-2} \nabla u \nabla v-\int_{D} f v,
$$

with the energy "norm" $\|v\|_{V(D)}^{p}=\int_{D} \kappa(x)|\nabla v|^{p}$. Denote

$$
I^{\omega_{i}} u=\arg \min _{v \in V^{c}\left(\omega_{i}\right)} \int_{\omega_{i}} \kappa(x)|\nabla u-\nabla v|^{2}, \forall u \in V^{h}\left(\omega_{i}\right) .
$$

Theorem 6.1. The main problem is solvable.

Sketch. Due to a page limit, we provide only a sketch of the proof here. After solving the sub-problem, we only need to establish the first inequality in the main problem. Since norms on $V^{c}\left(\omega_{i}\right)$ are equivalent, by the definition (6.2) of $I^{\omega_{i}}$ and the Hölder inequality, the inequality can be proved first on a subset of $V^{c}\left(\omega_{i}\right)$ where $\kappa(x)=c$. We show that the constants involved have upper bounds depending on $\operatorname{dim}\left(V^{c}\left(\omega_{i}\right)\right)$ but independent of $u$ and $\kappa$. The inequality can be proved next on $V^{c}\left(\omega_{i}\right)$ for a piecewise constant $\kappa$ with a corresponding partition of $V^{c}\left(\omega_{i}\right)$, and finally on $V^{c}\left(\omega_{i}\right)$ for general $\kappa$ by a limiting process.

In order to solve equation (6.1) we employ a Picard iteration such that

$$
-\operatorname{div}\left(\kappa(x)\left|\nabla u_{n-1}\right|^{p-2} \nabla u_{n}\right)=f \quad \text { for } x \in D,
$$

where the subscript $n$ denotes the iteration index. Since $p \geq 2$, we have $w_{0}^{1, p}(D) \subset W_{0}^{1,2}(D)=H_{0}^{1}(D) \subset W_{0}^{1, q}(D)$ and $H_{0}^{1}(D)$ is dense in $W_{0}^{1, q}(D)$, for numerical computation we use the variational form of equation (6.3)

$$
a_{n-1}\left(u_{n}, v\right)=f(v) \text { for all } v \in H_{0}^{1}(D),
$$

with usual bilinear forms. This equation has a matrix form $A_{n-1} u_{n}=b$, where for all $u_{n}, v \in V^{h}(D)$ we have

$$
u_{n}^{T} A_{n-1} v=\int_{D} \kappa\left|\nabla u_{n-1}\right|^{p-2} \nabla u_{n} \nabla v \quad \text { and } \quad v^{T} b=\int_{D} f v
$$

A solution is taken as $u:=u_{N}$ when $\left\|u_{N}-u_{N-1}\right\|$ (in some norm) is sufficiently small.

\subsection{The $p$-Laplacian: multiscale method}

Using the coarse mesh $\mathcal{T}^{H}$ we introduce coarse basis functions $\left\{\Phi_{i}\right\}_{i=1}^{N_{c}}$, where $N_{c}$ denotes the number of coarse bases. In this paper the basis functions are supported in the $\omega_{i}$ neighborhoods, however, an important consideration is that there may be multiple basis functions for each $\omega_{i}$. Given these coarse scale basis functions $\Phi_{i}$, we define the coarse-grid operator by $A_{0, n-1}=R_{0} A_{n-1} R_{0}^{T}$ where

$$
R_{0}^{T}=\left[\Phi_{1}, \ldots, \Phi_{N_{c}}\right] .
$$




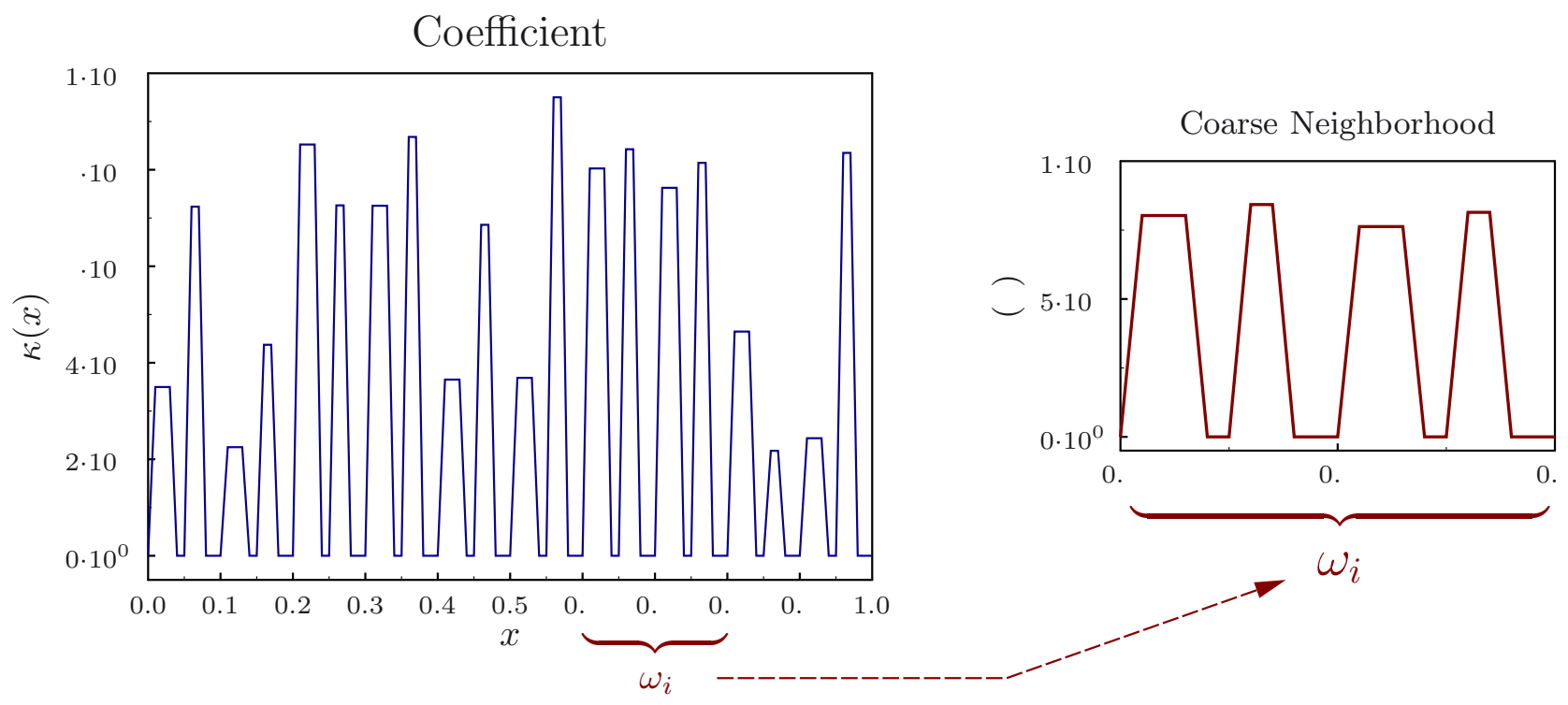

Figure 2. A high-contrast coefficient posed on a 100 element fine mesh (left); a coarse neighborhood from a 10 element coarse mesh (right).

In the expression above, the $\Phi_{i}^{\prime} s$ denote coarse-scale basis functions defined on a fine grid. For the discrete problem, they are simply vectors. Given the coarse space, we define the multiscale finite element solution as the finite element projection of the fine-scale solution into the coarse space $V^{C}$. In particular, the multiscale iterates are obtained by solving

$$
A_{0, n-1} u_{0, n}=f_{0},
$$

where $f_{0}=R_{0}^{T} b$. Equivalently, one may write the multiscale approximation on the coarse grid as $u_{0, n}=$ $\sum_{i} c_{i} \Phi_{i}$, where the $c_{i}$ are obtained through the variational form $a_{n-1}\left(u_{0, n}, v\right)=(f, v)$. We note that once $u_{0}$ is determined (i.e., when the coarse-scale Picard iteration converges), we have access to the corresponding fine-scale approximation of the solution through a basis reconstruction.

\subsection{Eigenvalue computations}

In this subsection we offer some results from the proposed non-linear eigenpair algorithm found in Section 4. To begin, we present Figure 2 as a representative example of a high-contrast coefficient $\kappa(x)$. See the left hand side of Figure 2 for an illustration of the coefficient defined on a global computational domain $D=[0,1]$. For the examples in this section, $\kappa(x)$ is posed on a global mesh with 100 fine elements, and the coarse discretization is chosen to contain 10 elements. The coefficient has a minimum value of $\kappa_{\min }(x)=1$ and the values in high-contrast regions are constructed from the uniform distribution $\mathcal{U}\left[10^{4}, 10^{5}\right]$. In addition, the coefficient is constructed such that 2 high-contrast regions occur in each coarse element (or equivalently, 4 high-contrast regions per coarse neighborhood). See the right hand size of Figure 2 for an illustration of a coarse neighborhood. We note that a fixed coefficient sample (resulting from the random generation above) is used for the numerical results in this section.

Throughout this section it is important to note that although the analysis from Section 4 requires an eigenpair correction for the cases when $p>2$, the predicted values suffice for the target application in this paper. Since the the two-level max-min procedure in the correction step requires a relatively large number of total iterations to ensure convergence, we choose the more efficient alternative of omitting the correction step. Of course, this 

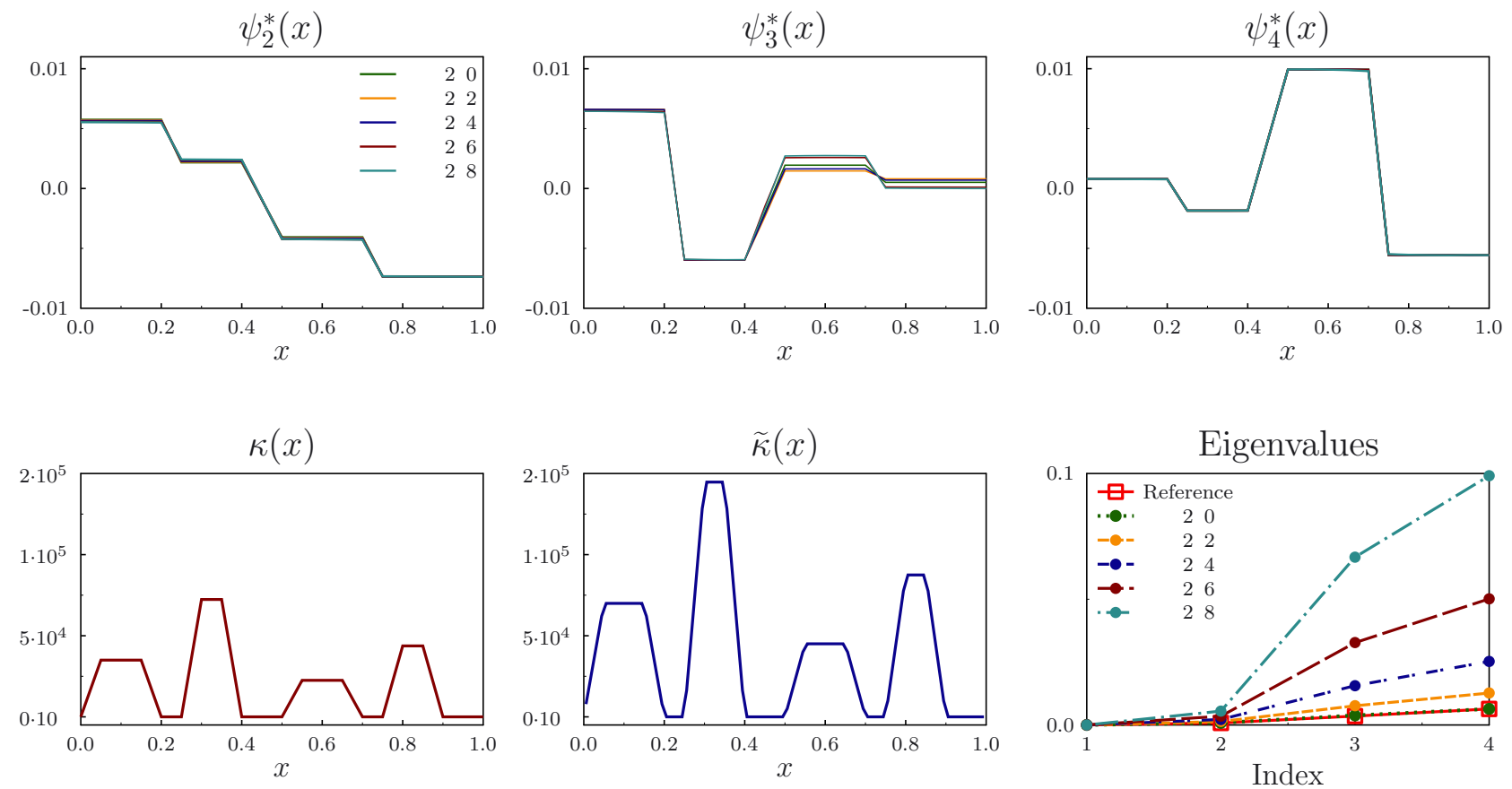

FiguRE 3. Eigenfunctions corresponding to a high contrast coefficient for $2.0 \leq p \leq 2.8$ (top); high contrast coefficients and eigenvalues (bottom).

gain in efficiency must occur without a significant compromise in the accuracy of the computed eigenpairs. To formally validate this choice we first note that the convergence criteria $\lambda^{\prime}\left(\psi^{*}\right)=0$ is equivalent to $\left\|w_{n}\right\| \rightarrow 0$. As such, if we ensure that $\left\|w_{n}\right\|$ is sufficiently small after the prediction step of the PCMM algorithm we can be confident that our computed eigenpairs closely capture the "exact" values. For this purpose, we use a convergence criterion such that $\left\|w_{n}\right\|<h$ where $h$ is the fine mesh size. For the examples in this paper the algorithm typically exits when $\left\|w_{n}\right\| \approx \mathcal{O}\left(10^{-3}\right)$, which is one order of magnitude smaller than our fine mesh. A max-min correction or a functional Newton's method may be used for more stringent convergence, however, detailed convergence results regarding the proposed algorithm will likely constitute a future publication.

We offer Figure 3 as an example of the eigenfunction/eigenvalue behavior corresponding to a weight coefficient $\kappa(x)$ and energy coefficient $\widetilde{\kappa}(x)$ (such as used in (4.8)). For this particular example, we limit ourselves to a fixed neighborhood $\omega_{i}$ with a rescaled horizontal axis. We use the algorithm in Section 4 with numerous values of $p$ ranging from $2.0 \leq p \leq 2.8$ and plot three eigenfunctions $\psi_{k}^{*}(x)$ for $k=2,3,4$ in the top row, along with the coefficients and corresponding eigenvalues in the bottom row. The figure shows that the eigenfunction behavior is quite similar for varying $p$ values. As expected, we see that the eigenfunctions are constant in the high contrast regions of the field and maintain the imposed zero-Neumann boundary conditions. In addition, we note that the computed eigenvalues from the PCMM algorithm for the case when $p=2.0$ are nearly indistinguishable from those obtained from a standard linear eigenpair computation. These results serve as further validation that prediction step of the non-linear algorithm yields suitable eigenpairs for the examples herein.

\subsection{Enriched multiscale solutions}

In this subsection we present a number of results verifying the enrichment procedure described in Section 3. To begin, we offer a series of fully resolved solutions to equation (6.1) in Figure 4. The solutions are obtained through solving the equation on a one-dimensional domain $D=[0,1]$ with zero Dirichlet conditions using 


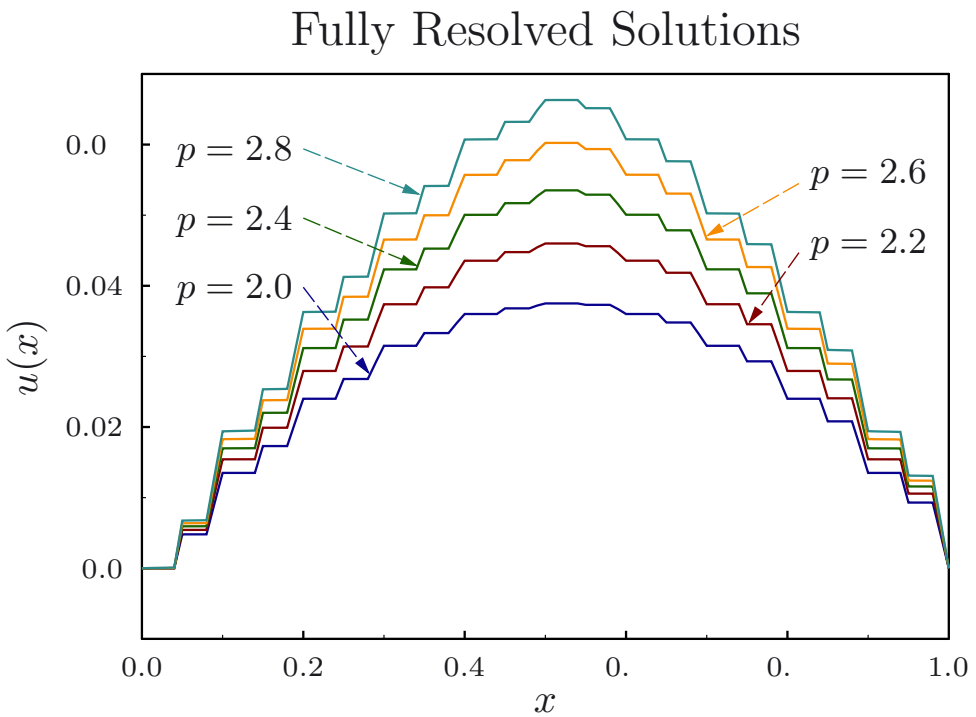

Figure 4. Solutions to (6.1) for $p=2.0,2.2,2.4,2.6,2.8$.

a variety of $p$ values such that $2.0 \leq p \leq 2.8$. We note that the solutions in Figure 4 are the benchmarks for our multiscale error comparisons. In order to construct the coarse space we start with an initial coarse space $V_{0}^{\text {initial }}=\operatorname{span}\left\{\chi_{i}\right\}$, where $\left\{\chi_{i}\right\}_{i=1}^{N_{c}}$ is a partition of unity subordinated to the covering $\left\{\omega_{i}\right\}$ such that $\chi_{i} \in V^{h}(D)$. We define the summed, pointwise energy $\widetilde{\kappa}$ as

$$
\widetilde{\kappa}=\kappa \sum_{i} H^{p}\left|\nabla \chi_{i}\right|^{p}
$$

and solve the Neumann eigenvalue problem (motivated by Sect. 3.2) using the algorithm from Section 4 in each coarse neighborhood $\omega_{i}$. We denote the non-linear eigenvalues and eigenvectors by $\left\{\lambda_{l}^{\omega_{i}}\right\}$ and $\left\{\psi_{l}^{\omega_{i}}\right\}$, respectively. We then define the set of coarse basis functions as by choosing $L_{i}$ eigenvectors that correspond to leading eigenvalues. We would like to note that when the nonlinearities depend on time, one needs to update the local eigenvalue problem.

For the numerical results we consider two sets of partitions of unity $\left\{\chi_{i}\right\}$ in which the enrichment procedure will be employed. In particular, we use a set of linear functions $\left\{\chi_{i}^{0}\right\}$ as well as a set of standard multiscale basis functions $\left\{\chi_{i}^{\mathrm{ms}}\right\}$ as initial partitions of unity. See the left hand side of Figure 5 for an illustration of a linear partition of unity, and the right hand side for a multiscale partition of unity.

For the comparisons in this section we use the relative energy error

$$
\left\|u-u_{\mathrm{ms}}\right\|_{V(D)} /\|u\|_{V(D)} \times 100 \%
$$

where $u$ denotes the fully resolved solution and $u_{\text {ms }}$ denotes a multiscale solution computed within an enriched multiscale space. For the tables we use notation of the form MsFEM $+m$, where $m$ denotes the number of additional basis functions that are used in the coarse space construction. For example, MsFEM+2 denotes a coarse space where $L_{i}=3$ total basis functions are used. While a linear partition of unity yields an understandably crude approximation to the fine scale solution (the errors are typically larger than $50 \%$ ), we note that the errors do indeed decrease as we include more eigenfunctions in the coarse space construction. We also refer back to Figure 3 and emphasize that when a linear partition of unity is used, $\widetilde{\kappa}$ has 4 inclusions and 4 channels within each coarse neighborhood. Thus, the fact that we obtain 4 small eigenvalues on each coarse neighborhood is consistent with what we expect from the the Raleigh Quotient. 

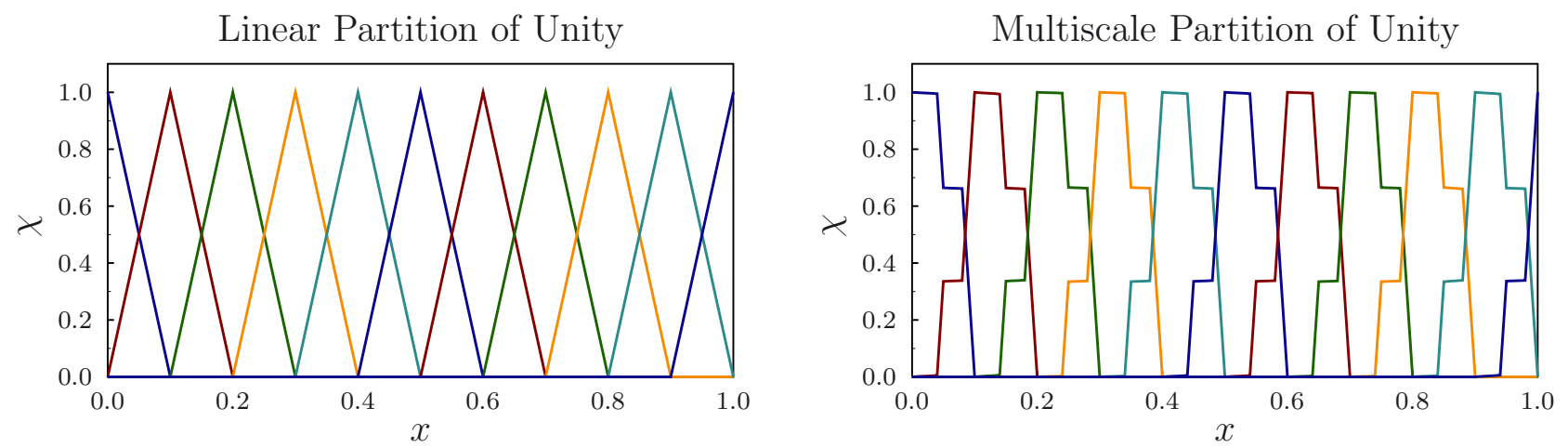

FiguRE 5. Linear partition of unity (left); multiscale partition of unity (right).

TABLE 1. Energy errors for a variety of $2.0 \leq p \leq 2.8$ and enriched coarse spaces constructed from a multiscale partition of unity.

\begin{tabular}{|c|c|c|c|c|c|}
\hline$p$ & 2.0 & 2.2 & 2.4 & 2.6 & 2.8 \\
\hline & \multicolumn{5}{|c|}{ relative energy error (\%) } \\
\hline MsFEM+0 & 7.50 & 7.02 & 6.80 & 6.77 & 6.97 \\
\hline $\mathrm{MsFEM}+1$ & 7.37 & 6.95 & 6.75 & 6.73 & 6.94 \\
\hline $\mathrm{MsFEM}+2$ & 0.62 & 0.36 & 0.49 & 0.98 & 1.76 \\
\hline $\mathrm{MsFEM}+3$ & 0.05 & 0.17 & 0.41 & 0.89 & 1.75 \\
\hline $\mathrm{MsFEM}+4$ & 0.04 & 0.11 & 0.29 & 0.62 & 1.14 \\
\hline
\end{tabular}

Aside from the linear case above, we are particularly interested in computing multiscale solutions that result from a multiscale partition of unity. That is, a partition of unity that is obtained through a process of computing localized basis functions in which we use the original global operator on each coarse subdomain (see Eq. (3.1)). See Table 1 for a variety of relative errors resulting from the enrichment procedure. We note that the initial basis set offers a more accurate solution due to the fact that the initial partition of unity is obtained through a series of localized solves (as opposed to simply assuming linear behavior). More importantly, we see that the errors significantly decrease as we include more bases in the multiscale space construction. Table 1 shows a situation where errors around 7\% may be decreased to values that are typically less than $1 \%$ when 4 basis additional basis functions are used in each coarse neighborhood. In particular, as we include more basis functions in the enriched space, we encounter a noticeable error decline in the multiscale solution. At this point we also consider the quantities that govern the error bounds that are presented in Appendix A. See Table 2 for a variety of $\min _{\omega_{i}} \lambda_{L_{i}+1}^{\omega_{i}}$ values as used in the analysis in the Appendix. We note that as $L_{i}$ increases, the eigenvalues increase, and the bound in equation (A.8) will correspondingly decrease. In other words, the analysis suggests (and the results validate) that keeping more basis functions for the coarse space construction will indeed yield a decreasing global error. In addition, we consider the effect that the coarse mesh size $H$ has on the convergence. The analysis in the Appendix (see, e.g. (A.8) also suggests that a decrease in $H$ will yield smaller errors. To validate this result, we offer Table 3 to for a comparison of energy errors obtained for the case when $H=0.1$ (the coarse mesh size used throughout the bulk of the results), as well as for the case when $H=0.05$ is refined. As the analysis suggests, we see a noticeable decline in the errors when a finer coarse mesh is used for the computations. Furthermore, we estimate the convergence rate (from (A.8)) to lie within the range $2.0 \leq l / 2 \leq 2.6$. We note that these values are recovered through computing the slope(s) from the associated log-log plot of the mesh size versus error when the $\Lambda_{*}$ values are comparable. 
TABLE 2. Scaled $\min _{\omega_{i}} \lambda_{L_{i}+1}^{\omega_{i}}$ values as described in Appendix A for a variety of $2.0 \leq p \leq 2.8$ and coarse basis configurations constructed from a multiscale partition of unity.

\begin{tabular}{lccccc}
\hline$p$ & 2.0 & 2.2 & 2.4 & 2.6 & 2.8 \\
\hline \multicolumn{5}{c}{$\min _{\omega_{i}} \lambda_{L_{i}+1}^{\omega_{i}}\left(\times H^{-p}\right)$} \\
\hline MsFEM+0 & $1.26 \mathrm{e} 2$ & $2.18 \mathrm{e} 2$ & $3.74 \mathrm{e} 2$ & $6.37 \mathrm{e} 2$ & $1.07 \mathrm{e} 3$ \\
MsFEM+1 & $5.40 \mathrm{e} 2$ & $1.07 \mathrm{e} 3$ & $2.10 \mathrm{e} 3$ & $4.10 \mathrm{e} 3$ & $7.93 \mathrm{e} 3$ \\
MsFEM+2 & $1.35 \mathrm{e} 3$ & $2.84 \mathrm{e} 3$ & $5.96 \mathrm{e} 3$ & $1.24 \mathrm{e} 4$ & $2.59 \mathrm{e} 4$ \\
MsFEM+3 & $2.70 \mathrm{e} 3$ & $6.38 \mathrm{e} 3$ & $1.50 \mathrm{e} 4$ & $3.50 \mathrm{e} 4$ & $8.09 \mathrm{e} 4$ \\
MsFEM+4 & $4.44 \mathrm{e} 3$ & $1.06 \mathrm{e} 4$ & $2.54 \mathrm{e} 4$ & $6.02 \mathrm{e} 4$ & $1.42 \mathrm{e} 5$ \\
\hline
\end{tabular}

TABle 3. Energy error values as described in Appendix A for a $H=0.05,0.1$ and coarse basis configurations constructed from a multiscale partition of unity.

\begin{tabular}{|c|c|c|c|c|c|}
\hline$p$ & 2.0 & 2.2 & 2.4 & 2.6 & 2.8 \\
\hline$H=0.1$ & \multicolumn{5}{|c|}{ relative energy error (\%) } \\
\hline $\mathrm{MsFEM}+0$ & 7.50 & 7.02 & 6.80 & 6.77 & 6.97 \\
\hline MsFEM+1 & 7.37 & 6.95 & 6.75 & 6.73 & 6.94 \\
\hline $\mathrm{MsFEM}+2$ & 0.62 & 0.36 & 0.49 & 0.98 & 1.76 \\
\hline$H=0.05$ & \multicolumn{5}{|c|}{ Relative energy error (\%) } \\
\hline $\mathrm{MsFEM}+0$ & 1.42 & 1.35 & 1.38 & 1.69 & 2.53 \\
\hline $\mathrm{MsFEM}+1$ & 0.07 & 0.22 & 0.53 & 1.10 & 2.04 \\
\hline $\mathrm{MsFEM}+2$ & 0.03 & 0.06 & 0.15 & 0.26 & 0.40 \\
\hline
\end{tabular}

TABLE 4. Convergence results for the domain decomposition algorithm: multiscale partition of unity.

\begin{tabular}{lccccc}
\hline$p$ & 2.0 & 2.2 & 2.4 & 2.6 & 2.8 \\
\hline MsFEM+1 & \multicolumn{5}{c}{ Number } \\
\hline$\eta_{\max }=10^{4}$ & 44 & 49 & 53 & 58 & 62 \\
$\eta_{\max }=10^{5}$ & 44 & 49 & 53 & 58 & 62 \\
$\eta_{\max }=10^{6}$ & 44 & 49 & 53 & 58 & 62 \\
\hline
\end{tabular}

To finish this section, we consider the domain decomposition algorithm in Section 5. In particular, we treat an enriched multiscale solution $u_{\mathrm{ms}}$ as a domain decomposition preconditioner and consider the convergence of the algorithm. See Table 4 for a variety of convergence results for the algorithm. We note that a stopping criterion of

$$
\left\|A u_{\mathrm{ms}}^{\mathrm{stop}}-f\right\|_{l^{q}}^{q} /\left\|A u_{\mathrm{ms}}^{\mathrm{init}}-f\right\|_{l^{q}}^{q}<10^{-4},
$$

where $\|\cdot\|_{l q}$ denotes the discrete $l_{q}$ norm with $1 / p+1 / q=1$, is used to assess convergence of the domain decomposition algorithm. In particular, we require that the initial residual is reduced by a factor of $10^{-4}$ for convergence. The results in Table 4 correspond to three separate contrast configurations. In particular, we recall that $\kappa_{\max }(x) / \kappa_{\min }(x)=\eta$, where $\eta$ is assumed to be large. The benchmark example throughout this section uses a coefficient where $\eta_{\max }=10^{5}$ (refer back to Fig. 2), and we construct two related coefficients where the contrast is both increased and decreased by the same order. From Table 4 we see in all cases that 62 or less iterations are required for the domain decomposition algorithm to reach convergence. Furthermore, the numerical results show that the number of iterations required to reach convergence do not depend on the contrast of the problem. However, an increase of $p$ does require more iterations for convergence. While, in general, we expect that varying the contrast will affect the iterative convergence rates, these results suggest that the domain decomposition procedure is independent of contrast for this problem. 


\section{Concluding Remarks}

In this paper, we developed multiscale finite element methods (MsFEM) and domain decomposition techniques for a class of nonlinear elliptic problems with high-contrast coefficients. In doing so, we extended existing work on linear problems to treat a class of nonlinear elliptic operators. As the systematic coarse space enrichment requires the solutions of a nonlinear eigenvalue problem, a detailed method for computing nonlinear eigenvalues was introduced. Convergence of the method was shown to relate to the dimension of the coarse space as well as a change in the coarse mesh size. We also showed that the coarse mesh spaces can be effectively used in two-level domain decomposition preconditioners, and a number of representative numerical results were offered to complement the analysis. In the future we hope to address more rigorous convergence properties of nonlinear eigenvalue algorithms, as well as apply the proposed method to time dependent problems and equations with random coefficients.

\section{APPENDix A}

\section{A.1 Proofs}

Proof. (Thm. 4.1)

By CFW (4.4), Sub-problem (4.2) is equivalent to

$$
\lambda_{k}^{\omega_{i},-}=\max _{A\left(\omega_{i}\right) \in \mathcal{S}_{k}\left(\omega_{i}\right)} \min _{u \in A_{S}\left(\omega_{i}\right)} \lambda^{\omega_{i}}(u), \quad k=2, \ldots, n .
$$

Since $V^{h}\left(\omega_{i}\right)$ is finite-dimensional, $A_{S}\left(\omega_{i}\right)$ is compact. Thus $G^{\omega_{i}}(u)$ attains its maximum and $G_{\chi}^{\omega_{i}}(u)$ attains its nonzero minimum on $A_{S}\left(\omega_{i}\right)$ and we have the inequalities

$$
\lambda_{1}^{\omega_{i}} \leq \lambda_{k}^{\omega_{i},-}=\max _{A\left(\omega_{i}\right) \in \mathcal{S}_{k}\left(\omega_{i}\right)} \min _{u \in A_{S}\left(\omega_{i}\right)} \lambda(u) \leq \frac{\max _{u \in B_{S}\left(\omega_{i}\right)} G^{\omega_{i}}(u)}{\min _{u \in B_{S}\left(\omega_{i}\right)} G_{\chi}^{\omega_{i}}(u)}<+\infty, \text { for all } k=2, \ldots, n .
$$

For each $k$, there are $\left\{A_{m}\left(\omega_{i}\right)\right\} \subset \mathcal{S}_{k}\left(\omega_{i}\right), \psi_{m}^{\prime, \omega_{i}}=\arg \min _{u \in\left[A_{m}\left(\omega_{i}\right)\right]_{S}} \lambda^{\omega_{i}}(u)$, such that $\lambda^{\omega_{i}}\left[\psi_{m+1}^{\prime \prime \omega_{i}}\right]>\lambda^{\omega_{i}}\left[\psi_{m}^{\prime, \omega_{i}}\right]$. Since $\left\{\psi_{i}^{\prime, \omega_{i}}\right\} \subset B_{S}\left(\omega_{i}\right)$, there are $\psi_{k}^{*, \omega_{i}} \in B_{S}\left(\omega_{i}\right),\left\{\psi_{m_{n}}^{\prime, \omega_{i}}\right\} \subset\left\{\psi_{m}^{\prime, \omega_{i}}\right\}$ such that $\psi_{m}^{\prime, \omega_{i}} \rightarrow \psi_{k}^{*, \omega_{i}}$ with

$$
\psi_{k}^{*, \omega_{i}}=\arg \max _{A\left(\omega_{i}\right) \in \mathcal{S}_{k}\left(\omega_{i}\right)} \min _{u \in A_{S}\left(\omega_{i}\right)} \lambda^{\omega_{i}}(u)=\arg \min _{u \in A_{k}^{\prime}\left(\omega_{i}\right)} \lambda^{\omega_{i}}(u) .
$$

Thus the solvability of (4.4) is established.

We next set $\lambda_{k}^{\omega_{i}}=\lambda\left(\psi_{k}^{*, \omega_{i}}\right)$ and note that we have $\lambda_{k}^{\omega_{i}}<\lambda_{k+1}^{\omega_{i}}$. We let $k_{0}$ be the smallest $k$ such that $\lambda_{k_{0}}^{\omega_{i}} \geq \lambda^{*, \omega_{i}}$. Then

$$
\lambda^{\omega_{i}}(u) \geq \lambda_{k_{0}}^{\omega_{i}} \geq \lambda^{*, \omega_{i}} \quad \text { or } \quad G^{\omega_{i}}(u) \geq \lambda^{*, \omega_{i}} G_{\chi}^{\omega_{i}}(u), \quad \text { for all } u \in A_{k_{0}}^{\prime}\left(\omega_{i}\right)
$$

So the solvability of the sub-problem is established.

\section{A.2 Convergence of MsFEM}

We write in each $\omega_{i}$

$$
-\operatorname{div}\left(\kappa(x)\left|\nabla\left(u-I^{\omega_{i}} u\right)\right|^{p-2} \nabla\left(u-I^{\omega_{i}} u\right)\right)=g,
$$

where $g$ is the residual in the approximation and $I^{\omega_{i}} u$ is the local approximant in $\omega_{i}$. The assumption on $g$ will be imposed later on. Multiplying both sides of (A.3) by $\chi_{i}^{p}\left(u-I^{\omega_{i}} u\right)$ and re-arranging the terms, we have

$$
\int_{\omega_{i}} \kappa \chi_{i}^{p}\left|\nabla\left(u-I^{\omega_{i}} u\right)\right|^{p} \leq \frac{C}{\delta} \int_{\omega_{i}} \kappa\left|\nabla \chi_{i}\right|^{p}\left|\left(u-I^{\omega_{i}} u\right)\right|^{p}+C \delta \int_{\omega_{i}} \kappa \chi_{i}^{p}\left|\nabla\left(u-I^{\omega_{i}} u\right)\right|^{p}+\left|\int_{\omega_{i}} g \chi_{i}^{p}\left(u-I^{\omega_{i}} u\right)\right|,
$$


where $C$ is independent of contrast. From here, we get Caccioppoli inequality

$$
\int_{\omega_{i}} \kappa \chi_{i}^{p}\left|\nabla\left(u-I^{\omega_{i}} u\right)\right|^{p} \preceq \int_{\omega_{i}} \kappa\left|\nabla \chi_{i}\right|^{p}\left|\left(u-I^{\omega_{i}} u\right)\right|^{p}+\left|\int_{\omega_{i}} g \chi_{i}^{p}\left(u-I^{\omega_{i}} u\right)\right| .
$$

Next, taking into account that MsFEM solution, $u_{H}$, provides a minimal energy, we have

$$
\begin{array}{r}
\int_{D} \kappa\left|\nabla\left(u-u_{H}\right)\right|^{p}=\int_{D} \kappa\left|\nabla\left(\sum_{i} \chi_{i}\left(u-I^{\omega_{i}} u\right)\right)\right|^{p} \preceq \sum_{i} \int_{\omega_{i}} \kappa\left|\nabla \chi_{i}\right|^{p}\left|u-I^{\omega_{i}} u\right|^{p} \\
+\sum_{i} \int_{\omega_{i}} \kappa\left|\chi_{i}\right|^{p}\left|\nabla\left(u-I^{\omega_{i}} u\right)\right|^{p} \preceq \sum_{i} \int_{\omega_{i}} \kappa\left|\nabla \chi_{i}\right|^{p}\left|u-I^{\omega_{i}} u\right| p+\left.\sum_{i}\left|\int_{\omega_{i}} g\right| \chi_{i}\right|^{p}\left(u-I^{\omega_{i}} u\right)
\end{array}
$$

Using the fact that $\int_{\omega_{i}} \sum_{i} \kappa\left|\nabla \chi_{i}\right|^{p}\left(u-I^{\omega_{i}} u\right)^{p} \preceq \frac{1}{\lambda_{L_{i}+1}^{\omega_{i}}} \int_{\omega_{i}} \kappa\left|\nabla\left(u-I^{\omega_{i}} u\right)\right|^{p}$, we have

$$
\begin{aligned}
& \sum_{i} \int_{\omega_{i}} \kappa\left|\nabla \chi_{i}\right|^{p}\left|u-I^{\omega_{i}} u\right|^{p} \preceq \sum_{i} \int_{\omega_{i}} \sum_{i} \kappa\left|\nabla \chi_{i}\right|^{p}\left|u-I^{\omega_{i}} u\right|^{p} \\
\preceq & \sum_{i} \frac{1}{\lambda_{L_{i}+1}^{\omega_{i}}} \int_{\omega_{i}} \kappa\left|\nabla\left(u-I^{\omega_{i}} u\right)\right|^{p} \preceq \sum_{i} \frac{\alpha_{L_{i}+1}^{\omega_{i}}}{\lambda_{L_{i}+1}^{\omega_{i}}} \int_{\omega_{i}} \kappa \sum_{i}\left|\chi_{i}\right|^{p}\left|\nabla\left(u-I^{\omega_{i}} u\right)\right|^{p} \\
\preceq & \sum_{i} \frac{\alpha_{L_{i}+1}^{\omega_{i}}}{\lambda_{L_{i}}^{\omega_{i}}} \int_{\omega_{i}} \kappa\left|\chi_{i}\right|^{p}\left|\nabla\left(u-I^{\omega_{i}} u\right)\right|^{p} \\
\preceq & \sum_{i} \frac{\alpha_{L_{i}+1}^{\omega_{i}}}{\lambda_{L_{i}+1}^{\omega_{i}}} \int_{\omega_{i}} \kappa\left|\nabla \chi_{i}\right|^{p}\left|u-I^{\omega_{i}} u\right|^{p}+\left.\sum_{i} \frac{\alpha_{L_{i}+1}^{\omega_{i}}}{\lambda_{L_{i}+1}^{\omega_{i}}}\left|\int_{\omega_{i}} g\right| \chi_{i}\right|^{p}\left(u-I^{\omega_{i}} u\right) \mid \\
\preceq & \frac{1}{\Lambda_{*}}\left(\sum_{i} \int_{\omega_{i}} \kappa\left|\nabla \chi_{i}\right|^{p}\left|u-I^{\omega_{i}} u\right|^{p}+\left.\sum_{i}\left|\int_{\omega_{i}} g\right| \chi_{i}\right|^{p}\left(u-I^{\omega_{i}} u\right) \mid\right),
\end{aligned}
$$

where $\Lambda_{*}=\min _{\omega_{i}} \lambda_{L_{i}+1}^{\omega_{i}} / \alpha_{L_{i}+1}^{\omega_{i}}$ and $\alpha_{L_{i}+1}^{\omega_{i}}=\int_{\omega_{i}} \kappa\left|\nabla\left(u-I^{\omega_{i}} u\right)\right|^{p} / \int_{\omega_{i}} \kappa \sum_{i}\left|\chi_{i}\right|^{p}\left|\nabla\left(u-I^{\omega_{i}} u\right)\right|^{p}$ that represents the error concentration near the boundaries of $\omega_{i}$. One can use larger domains and oversampling strategies to control $\alpha_{L_{i}+1}^{\omega_{i}}$ (see [8] and references therein) that will be investigated in our future work. Applying this inequality $n$ times, we have

$$
\begin{aligned}
& \sum_{i} \int_{\omega_{i}} \kappa\left|\nabla \chi_{i}\right|^{p}\left|u-I^{\omega_{i}} u\right|^{p} \\
\preceq & \left(\frac{1}{\Lambda_{*}}\right)^{n} \sum_{i} \int_{\omega_{i}} \kappa\left|\nabla \chi_{i}\right|^{p}\left|u-I^{\omega_{i}} u\right|^{p}+\left.\sum_{j=1}^{n}\left(\frac{1}{\Lambda_{*}}\right)^{j} \sum_{i}\left|\int_{\omega_{i}} g\right| \chi_{i}\right|^{p}\left(u-I^{\omega_{i}} u\right) \mid \\
\preceq & \left(\frac{1}{\Lambda_{*}}\right)^{n} \sum_{i} \int_{\omega_{i}} \kappa\left|\nabla \chi_{i}\right|^{p}\left|u-I^{\omega_{i}} u\right|^{p}+\left(\Lambda_{*}\right)^{n}\left(\frac{1-\Lambda_{*}^{n}}{\Lambda_{*}-1}\right) \sum_{i} \int_{\omega_{i}}\left(|\kappa|\left|\nabla \chi_{i}\right|^{p}\right)^{-q / p} g^{q} .
\end{aligned}
$$

Considering $\sum_{i} \int_{\omega_{i}} \kappa\left|\nabla \chi_{i}\right|^{p}\left|u-I^{\omega_{i}} u\right|^{p} \preceq \sum_{i} \int_{\omega_{i}} \sum_{i} \kappa\left|\nabla \chi_{i}\right|^{p}\left|u-I^{\omega_{i}} u\right|^{p} \preceq \int_{D} \kappa|\nabla u|^{p}$, we have the following convergence rate for MsFEM

$$
\int_{D} \kappa\left|\nabla\left(u-u_{H}\right)\right|^{p} \preceq \frac{1}{\Lambda_{*}^{n+1}} \int_{D} \kappa\left|\nabla\left(u-I^{\omega_{i}} u\right)\right|^{p}+\left(\left(\Lambda_{*}\right)^{n}\left(\frac{1-\Lambda_{*}^{-n}}{\Lambda_{*}-1}\right)+1\right) R,
$$

where $R=\sum_{i} \int_{\omega_{i}}\left(|\kappa|\left|\nabla \chi_{i}\right|^{p}\right)^{-q / p} g^{q}$ is assumed to be bounded. 
Assumption. We assume that there exists a global function $G, \int_{D} G^{q} \leq C$ such that $\int_{\omega_{i}}\left(\kappa\left|\nabla \chi_{i}\right|^{p}\right)^{-q / p} g^{q} \preceq$ $H^{l} \int_{\omega_{i}} G^{q}$ Note that because $\nabla \chi_{i}$ behaves as $H^{-1}, l=q$ is an appropriate choice.

With this assumption, we have the following convergence rate for MsFEM.

$$
\int_{D} \kappa\left|\nabla\left(u-u_{H}\right)\right|^{p} \preceq \frac{1}{\Lambda_{*}^{n+1}} \int_{D} \kappa|\nabla u|^{p}+\left(\left(\Lambda_{*}\right)^{n}\left(\frac{1-\Lambda_{*}^{-n}}{\Lambda_{*}-1}\right)+1\right) H^{l} \int_{D}|G|^{p} .
$$

Choosing $\Lambda_{*}$ sufficiently large and assuming $\int_{D} \kappa|\nabla u|^{2}$ and $\int_{D} G^{2}$ are bounded and choosing in each $\omega_{i}, n=$ $-\frac{-l}{2} \frac{\log (H)}{\log \Lambda_{*}}$, we obtain

$$
\int_{D} \kappa\left|\nabla\left(u-u_{H}\right)\right|^{p} \preceq\left(\frac{H^{l / 2}}{\Lambda_{*}}\right)
$$

\section{REFERENCES}

[1] J. Aarnes, S. Krogstad and K. Lie, A hierarchical multiscale method for two-phase flow based on upon mixed finite elements and nonuniform coarse grids. SIAM Multiscale Model. Simul. 5 (2006) 337-363.

[2] T. Arbogast, G. Pencheva, M. Wheeler and I. Yotov, A multiscale mortar mixed finite element method. SIAM Multiscale Model. Simul. 6 (2007) 319-346.

[3] X. Cai and D. Keyes, Nonlinearly preconditioned inexact Newton algorithms. SIAM J. Sci. Comput. 24 (2002) 183-200.

[4] X. Chen and Y. Lou, Principal eigenvalue and eigenfunction of elliptic operator with large convection and its application to a competition model. Indiana Univ. Math. J. 57 (2008) 627-658.

[5] M. Dryja and W. Hackbusch, On the nonlinear domain decomposition method. BIT 37 (1997) $296-311$.

[6] Y. Efendiev, J. Galvis, R. Lazarov, S. Margenov and J. Ren, Robust two-level domain decomposition preconditioners for high-contrast anisotropic flows in multiscale media. Comput. Method Appl. Math. 12 (2012) 1-22.

[7] Y.Efendiev, J. Galvis and T. Hou, Generalized Multiscale Finite Element Method. J. Comput. Phys. (2013) 116-135.

[8] Y. Efendiev, J. Galvis, G. Li and M. Presho, Generalized Multiscale Finite Element Methods. Oversampling strategies. To appear in Int. J. Multiscale Comput. Engrg.

[9] Y. Efendiev, J. Galvis and X. Wu, Multiscale finite element methods for high-contrast problems using local spectral basis functions. J. Comput. Phys. 230 (2011) 937-955.

[10] J. Galvis and Y. Efendiev, Domain decomposition preconditioners for multiscale flows in high contrast media. SIAM Multiscale Model. Simul. 8 (2010) 1461-1483.

[11] Y. Efendiev and T. Hou, Multiscale Finite Element Methods: Theory and Applications. Springer, New York (2009).

[12] T. Hou and X. Wu, A multiscale finite element method for elliptic problems in composite materials and porous media. J. Comput. Phys. 134 (1997) 169-189.

[13] T. Hughes, G. Feijóo, L. Mazzei and J. Quincy, The variational multiscale method - a paradigm for computational mechanics. Comput. Methods Appl. Mech. Engrg. 166 (1998) 3-24.

[14] P. Jenny, S. Lee and H. Tchelepi, Multi-scale finite volume method for elliptic problems in subsurface flow simulation. J. Comput. Phys. 187 (2003) 47-67.

[15] T. Mathew, Domain Decomposition Methods for the Numerical Solution of Partial Differential Equations. BIT 37 (1997) 296-311.

[16] P. Solin and S. Giani, An iterative adaptive finite element method for elliptic eigenvalue problems. J. Comput. Appl. Math. 236 (2012) 4582-4599

[17] X. Tai and M. Espedal, Rate of convergence of some space decomposition methods for linear and nonlinear problems. SpringerVerlag, Berlin-Heidelburg (2008).

[18] J. Xu and L. Zikatanov, On an energy minimizing basis for algebraic multigrid methods. Comput. Visual Sci. 7 (2004) $121-127$.

[19] X. Yao and J. Zhou, Numerical methods for computing nonlinear eigenpairs. Part I. Iso-homogeneous cases. SIAM J. Sci. Comput. 29 (2007) 1355-1374.

[20] X. Yao and J. Zhou, Numerical methods for computing nonlinear eigenpairs. Part II. Non iso-homogenous cases. SIAM J. Sci. Comp. 30 (2008) 937-956.

[21] E. Zeidler, Nonlinear Functional Analysis and Its Applications III. Springer-Verlag, New York (1985). 